were prognostic for poor event-free and overall survival. In multivariate analysis, TNF-R1, IL1RA, IL-6 and sCD30 were all prognostic for poor outcome. A cytokine prognostic index comprising the three most discriminating factors, sCD30, IL-6 and IL1RA, was able to predict the outcome of patients with Hodgkin's lymphoma independently of three classical prognostic scoring systems. Moreover, the cytokine prognostic index was able to further stratify patients who were grouped according to the International Prognostic Score, identifying patients with an especially high probability of experiencing treatment failure.

The authors conclude that the plasma cytokine signature could identify patients with Hodgkin's lymphoma at high risk of firstline treatment failure, allowing physicians to adapt treatment accordingly.

Original article Casasnovas R-O et al. (2007) Plasma cytokine and soluble receptor signature predicts outcome of patients with classical Hodgkin's lymphoma: a study from the Group d'Etude des Lymphomes de l'Adulte. J Clin Oncol 25: $1732-1740$

\section{HLA-matched donor HSC allografts improve survival in newly diagnosed myeloma}

Chemotherapy and autologous hematopoietic stem cell (HSC) transplantation is a standard treatment for patients with newly diagnosed multiple myeloma; however, recurrences are often observed. Bruno at al. compared the outcomes of patients who received an HSC autograft followed by an HLA-matched allograft, with those of patients who received HSC autografts only.

The study included 162 patients with stage II or III myeloma who had at least one sibling. All patients received chemotherapy followed by melphalan and autologous stem cell transplantation. Patients were divided into two groups: those with HLA-identical siblings, who received HSC allografts (group $A$ ); and those with no HLA-identical sibling, who received a second autologous HSC transplantation (group B). Patients who received HSC allografts had a longer overall survival than did patients who received a second autologous HSC transplant (80 months vs 54 months; $P=0.01$ ). The median event-free survival was higher in patients with HLA-identical donors than in those without HLA-matched donors (35 months vs 29 months; $P=0.02$ ). The rate of complete remission was higher in group $A$ than in group $B$ (55\% vs 26\%; $P=0.004$ ). Disease-related mortality was significantly higher in group $B$ than in group A (43\% vs 7\%; $P<0.001)$. In group A, 21 patients $(36 \%)$ were in complete remission at a median follow-up of 38 months after allografting. By contrast, at 36 months after the second transplantation, only 4 of the 46 patients who received two autografts were in complete remission. The authors conclude that an HSC allograft after chemotherapy and an HSC autograft prolongs survival in patients with newly diagnosed myeloma.

Original article Bruno B et al. (2007) A comparison of allografting with autografting for newly diagnosed myeloma. N Engl J Med 356: 1110-1120

\section{Temozolomide is an active agent for the treatment of primary brain lymphomas}

Methotrexate-based chemotherapy is the firstline treatment for patients with primary brain lymphoma; however, in most cases this disease remains incurable. The treatment options for patients with recurrent primary brain lymphoma are limited, and so far there is little evidence to indicate that multi-agent chemotherapy is superior to methotrexate alone. Reni and coauthors have recently assessed the activity of the alkylating agent temozolomide in patients with relapsed or refractory primary brain lymphoma.

This phase II study included 36 patients with a median age of 60 years (range 34-81 years), who had been diagnosed with primary brain lymphoma and had previously failed to respond to high-dose methotrexate and/or radiotherapy treatment. The patients received $150 \mathrm{mg} / \mathrm{m}^{2}$ of temozolomide daily for 5 days every 4 weeks, for a maximum of six cycles. This salvage treatment yielded nine complete responses and two partial responses (response rate $31 \%$, 95\% Cl 16-46\%). The median overall survival was 3.9 months (interquartile range 1.716 months), and the median progression-free survival was 2.8 months (interquartile range 1-8 months). The 1-year overall survival was $31 \%$ (95\% Cl 16-46\%). The drug was generally well-tolerated, with two patients experiencing grade 4 neutropenia, and one patient 